\title{
Multi Mobile Agents for Multimedia File Download in E- Commerce Environment
}

\author{
Nirmala C R \\ Research Scholar \\ BIET, Davangere
}

\author{
Dr. V Ramaswamy \\ Prof \& Head \\ IS \& E Department \\ BIET, Davangere
}

\author{
Jyothi N M \\ Lecturer, MCA Dept \\ BIET, Davangere
}

\begin{abstract}
So far, the global standard for file uploading and downloading over internet has been FTP. A technology which is emerging fast on web today is mobile agent technology which can be applied for file download from remote server to remote client or server. We investigate the use of mobile agents for downloading of files from remote hosts. We believe this technology has the potential to foster new ways of downloading of files when compared to traditional FTP. This finds its application in achieving faster ecommerce, while purchasing the items online. We send mobile agent embedded within it the various types of file name/s to be downloaded from the remote server by specifying the itinerary details. The agent on reaching the server gets the file/s from the server and returns to the client along with the file. An e-commerce system of purchasing multimedia files (Audio, Video, and Image) online is implemented here. Simulation of this work has been carried out on IBM'S Aglets framework and the performance has been tested by computing the round trip time. We have also devised an algorithm for purchasing the products which are in restricted realm. This technique appears to be faster when compared to traditional client server FTP based file download system.
\end{abstract}

\section{General Terms}

Artificial Intelligence, E-commerce, Pull Model , Algorithm, Multimedia, File Transfer Protocol, Agent Transfer Protocol, Mirror Sites, TCP//IP, LAN.

\section{Keywords}

Multi Mobile Agents, Multimedia files, User Module, Web Module, Web Agent, User Agent, Data Size, Static Agent.

\section{INTRODUCTION}

Internet is both a powerful resource and a powerful challenge for applications. While it provides a great deal of information, it is also a dynamic resource of information which means old contents might disappear whereas new and better contents are continuously added. Users of web may be interested in downloading / uploading information. Web is the popular source of information retrieval.

One requirement for the e-commerce applications is that information should be uploaded at the faster rate and downloading also should be equally fast. Having said this, we are interested in discovering how mobile agents can fruitfully applied in place of traditional FTP and how mobile agents can be made to accelerate the speed of downloading. For example, if a user wants to buy a multimedia file like an audio track online, instead of using the FTP and its mirror sites, which are heavily loaded, the user can employ mobile agent to get the file from the desired remote server. This system follows the pull model of e-commerce.

The current approach to file download is based on FTP which suffers from a number of disadvantages. FTP is connection oriented and works very slowly if there is more traffic on the Internet. This reduces the speed of transfer of mega bytes per second. If Internet connection is disrupted, the file download will be cutoff causing more inconvenience to the users.

The proposed framework called "Multi Mobile Agent Mediated Multimedia File download in E-commerce Environment" demonstrates its effectiveness over traditional FTP. The main advantage of the framework is that it frees the remote client so that it can perform more essential tasks. The user can send / receive the file in which she / he is interested. The user does not have to worry about speed of Internet, Internet cutoffs and rate of transfer of megabytes per second. Thus this framework acts as an ideal frame work for e-commerce applications which require faster downloading of relatively smaller data up to 64 megabytes.

\section{MOBILE AGENTS}

"Mobile agents are processes dispatched from source computer to accomplish a specified task $[3,4]$. Each mobile agent is a computation along with its own data and execution state. After its submission, the mobile agent proceeds autonomously and independently of the sending client. When the agent reaches a server, it is delivered to an agent execution environment. If the agent possesses necessary authentication credentials, its executable parts are started. To accomplish its task, the mobile agent can transport itself to another server, spawn new agents, or interact with other agents. Upon completion, the mobile agent delivers the results to the sending client or to another server."

\section{"A broad definition of a Mobile Agent"}

Mobile Agents appeared in the scene during the last decade of the previous millennium. Researchers and practitioners look at Mobile Agent technology as a potential technology that could revolutionize the way we perform computations, develop applications and systems. They were, and still are, viewed as a unique way to approach mobile and wireless computing. Mobile agents have been used in a variety of applications and computing areas. The driving force which motivates mobile agent-based computation is multifold, Mobile agents provide an efficient, asynchronous method for searching for information or services in rapidly evolving network; Mobile agents may be launched into the unstructured network and made to roam around for gathering information. Mobile agents support intermittent connectivity, 
slow networks and light-weight devices. Thus, mobile agents provide several benefits in Internet system programming [1, 2, 4], in which there is a need for different kinds of integrated information, monitoring and notification, encapsulating artificial intelligence techniques, security and robustness [2,3,6]. Also the mobile agent paradigm has demonstrated satisfactory performance when deployed for distributed access to Internet databases, distributed retrieving and filtering of information and minimizing network workload $[1,3,4,56]$. Finally, mobile agents have proved very effective in supporting asynchronous execution of client requests, weak connectivity and disconnected operations and the dynamic adaptation to the various types of user connectivity common in wireless environments $[4,5$, and 6].

Various mobile agent platforms have been developed. They can be broadly categorized as Java and non-Java based ones, and they can be further split into experimental and commercial ones. There is an increasing interest in Java-based platforms due to the inherent advantages of Java namely platform independence support, highly secure program execution and small size of compiled code. These features of Java together with its simple database connectivity interface (JDBC) which facilitates application access to multiple relational databases over the Web make the Java approaches very attractive. In fact, this JAVA orientation of the Mobile Agent technology has given the much needed boost and has kindled the hope that this technology can do and offer much more.

\section{FILE TRANSFER PROTOCOL [RFC959]}

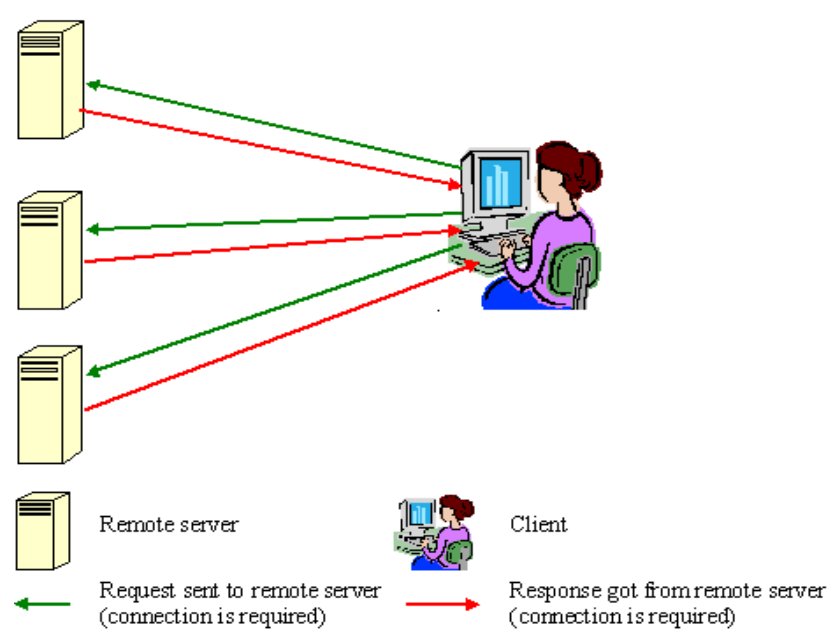

Figure.1. Architecture of FTP based File Download

FTP stands for File Transfer Protocol which is used for file download over the network. It transfers the data serially. It is connection oriented. FTP specifies the target address to which the file is to be downloaded or uploaded. Both source and destination addresses are specified. The transfer rate of the FTP is dependent on the network traffic. If more number of files are to be downloaded then they should be either zipped (compressed) and downloaded or should be downloaded serially one after the other. In this process, if the Internet connection is disrupted, the file download is cut off necessitating the download process to be restarted from the beginning once the connection is reestablished.
Of course some software are available using which the file downloading can be restarted from the cutoff point. In any case, if large size files are involved, the time taken by FTP is directly dependent on the size of the file, Internet connection and Internet traffic.

\subsection{Drawbacks of File Transfer Protocol}

Following are some of the drawbacks of using traditional FTP for the purpose of uploading and downloading of files.

- For transferring of files, source and destination should be in constant connection with each other.

- File transfer takes place sequentially.

- If the file size is more, more time is required to download.

- Large sized multiple files cannot be downloaded simultaneously.

- If the internet traffic is high, the data transfer rate becomes very low thus consuming more time.

\section{MOBILE AGENTS for FILE DOWNLOAD}

In order to accomplish the task of downloading the file, consider following scenario of buying multimedia content online. The architecture must consist of the following two subsystems which are called modules

\subsection{User Module}

The user module consists of four primary components namely User Agent (UA), a graphical User Interface, data structures containing unsatisfied file download requests from which instructions to the download mobile agents are generated, a data structure of pending download reservations and confirmations and a downloader agent which is mobile agent.

\subsection{Web Module.}

This module consists of a stationary agent called Web Agent, which is responsible for processing the request from any visiting downloader agent.
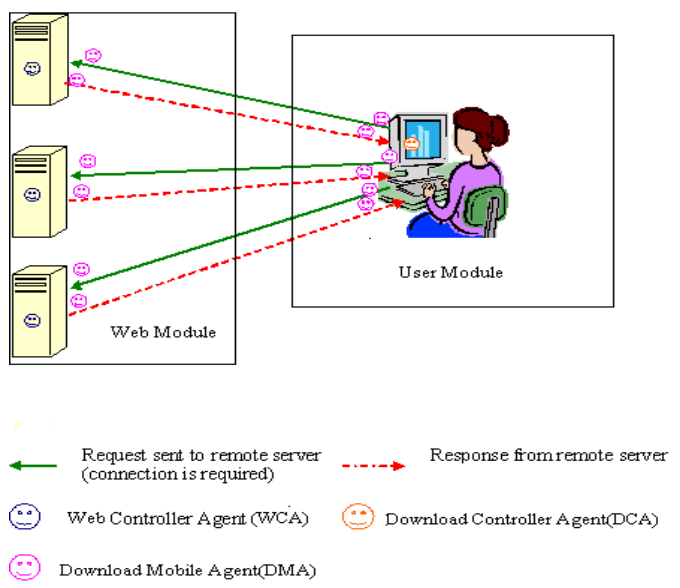

Figure 2. Mobile Agent Architecture For File Download From Remote Server

Today, we are in an era of Electronic-Commerce. Anything required is available at fingertips with the help of World Wide Web. All the web applications follow client/server architecture 
which has got many disadvantages. Mobile agents overcome these serious drawbacks of client/server architecture. Many ECommerce applications require the downloading and uploading of information, service and applications to the remote servers from the remote clients and this downloading will have to be carried out in real time. Hence we propose an architecture which is supposed to help in faster downloading. Use of mobile agents hastens the process downloading.

Mobile agents are being used for downloading file (irrespective of its type). These agents in turn make use of agent transfer protocol for the purpose of communication between hosts. Procedure to download file using mobile agents is as follows.

We assume that client and server are interchangeable which means that, whenever server wants to download some important file, it becomes client and the client will become the server.

Remote Client (user) downloads the file using User Agent (UA). UA is stationary agent at the User Module

- Remote client sends a request to the server using UA to download particular file which has file name/s specified in the downloader Agent (DA). The itinerary details for DA are predefined by the user which routes it to the remote server.

- Remote Server has Web Agent (WA). This agent is a stationary agent at the server side. On arrival of the DA, WA reads the requested file name/s in the DA (query) and searches for those files in its local directory and also executes query on the web databases maintained. If files are found, they are retrieved and attached to the DA. Otherwise returns "file not found".

- The DA after obtaining the file/s returns back to the remote client from where it was dispatched and hands over the requested file/s to UA which can be accessed by the user. The files can be stored in the database maintained at the client machine.

If more than one file is requested, DA can carry up to its maximum capacity. Multiple DAs could be dispatched by UA by using bee-hive [9] approach concurrently to bring the remaining files.

\section{EXPERIMENTAL SET UP}

\subsection{File Download Using FTP}

In order to download various types of files, the following set up is made in the laboratory. Networking is done using Linux, Windows, Macintosh and Sun Solaris 9.0 Operating Systems. File transfer protocol is configured on TCP/IP. All the hosts considered for this are connected to the different LANs. One host is considered from each network. Host A is on Linux Network, Host $\mathrm{B}$ is on Windows Network, Host $\mathrm{C}$ is on Macintosh Network, Host D is on Solaris Network and Host E is on ftp://www.bietdvg.edu residing at SPSintrard Systems, Bangalore. Karnataka. Assume that Host $\mathrm{A}$ is in need of downloading different types of files from B, C, D and E. Following observations are made.

In this set up, the dispatching server also acts as a remote server. Note that the request for download originated from server A. Following graph shows the observations made while downloading the files using File Transfer Protocol. Five files of type's .doc, .jpg, .wav, .html, and .pdf of varying sizes are downloaded from the different locations remotely. A small timer program was run to note down the request time and the download time. The total time
$T_{t}$ taken for downloading all the five files is given by $T_{t}=R_{t}+D_{t}$ $+R_{t d}$ where $R_{t}$ denotes Request Time, $D_{t}$ denotes Download time and $R_{t d}$ denotes Time between the requests.

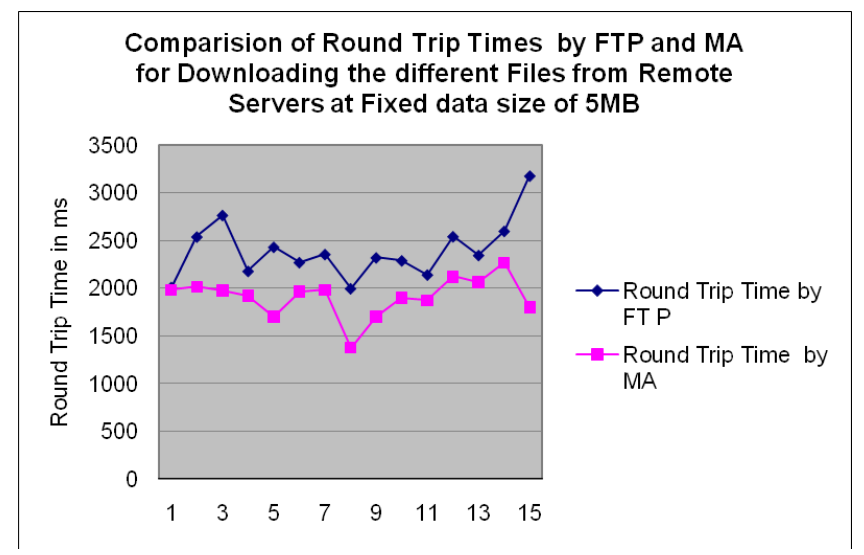

Figure 3. Graph Showing comparison of Round Trip Time for Downloading various files from remote Severs by both FTP and MA Technology with Fixed Data Size

From the Figure 3, we can observe that, the Round trip time taken by traditional client server FTP is more compared to Round trip time taken by MA technology

\subsection{File Download by Parallel Execution of Mobile Agents}

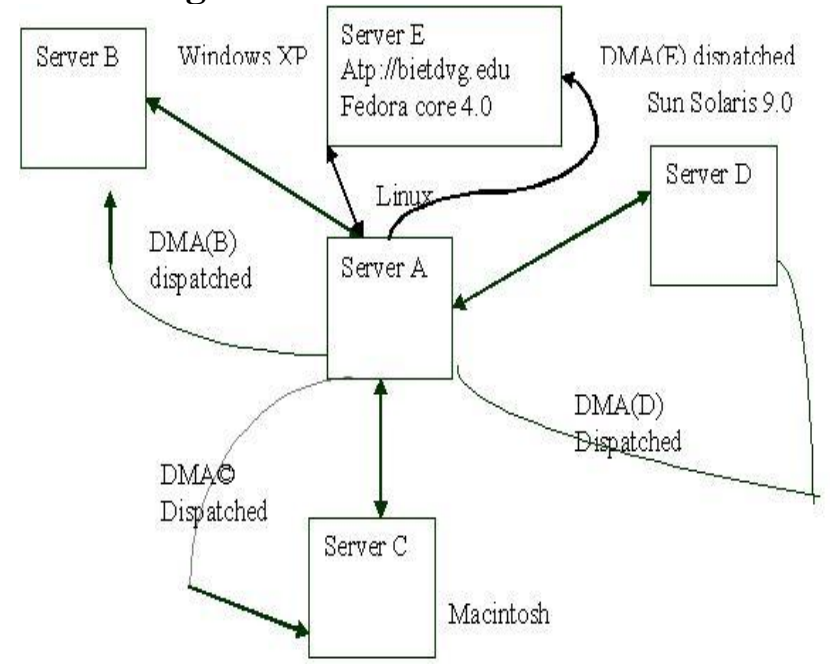

Figure 4. Setup Showing Parallel Execution of Mobile Agents for the purpose of downloading the files

Similar set up is used with IBM's Aglet Framework 2.0.2 installed on all the hosts from where the files have to be downloaded. The hosts are also installed with jdk1.5.0_18 version. The set up is shown in Figure 4. Each server is a web module that consists of static Web Agent. A is considered as the dispatching server, where the user module sits. Once the client wants to download the files, he can create any number of UAs and in turn the DAs[9]. As the file sizes chosen here are comparatively small, a single mobile agent can perform the job of getting all the files without over or under utilizing the capacity of the agent. 
Downloader Agent DA first searches and retrieves Test.doc from host $\mathrm{A}$ and then moves to host $\mathrm{B}$, appends Test1.jpg, then to $\mathrm{C}$ and retrieves spe.wav, later migrates to host $D$ and adds Mul.html and finally visits bietdvg.edu and appends its vector with Multi.pdf and finally travels back to dispatching Host A. The files collected from all the hosts are handed over to User Agent. The user module with the help of User Agent appends any messages from mobile agents travelling to the data structures for any pending and reservation confirmations. The Departure and Arrival timings of DA are recorded and round trip time taken is computed.

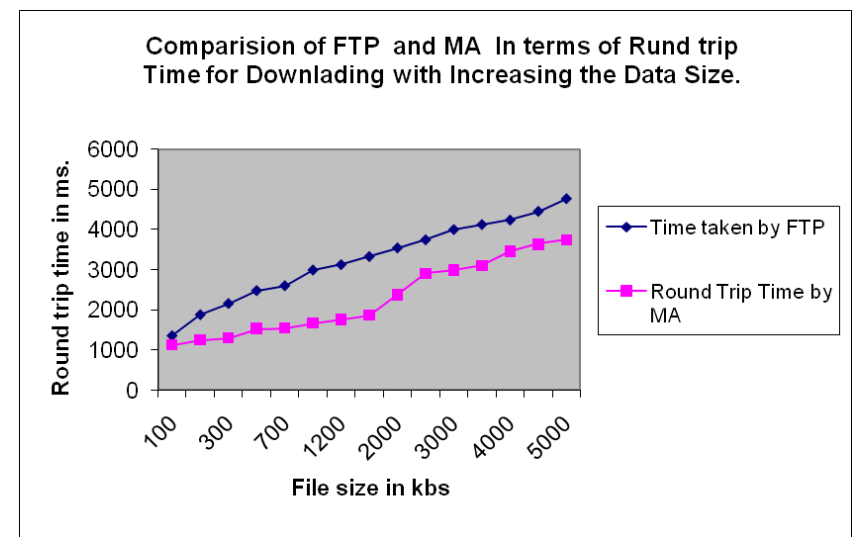

\section{Figure 5. Graph Showing the Time Comparison with Increasing Data size.}

Form Figure 5. We can observe that, for data sizes up to 64 MB's the performance of the mobile agent is more compared to the FTP based downloading. There is solution for downloading the large data size, i.e. more than one mobile agent can be employed.

The above scenario works for the case of free downloads from many hosts at free of cost. But most of the times, if anybody wants to buy any item like Audio, Video or some time books, journal papers and games etc, he/she has to make payment online and then download. Consider the example of buying audio track of Swami Vivekananda speech at Chicago, different domains may charge for the file differently. The mobile agent, which is responsible for downloading that file, has to compare for the price at different hosts by using the following algorithm.

1. The Downloader Agent (DA) when it visits every host defined in its itinerary, it transfers request to WA of remote host. WA processes the request and update DA with the result, now the comparison starts.

2. Downloader Agent takes two attributes as comparison metrics. One is name of the file and the second attribute is its price, both these attributes are predefined with the downloader agent.

3. With these predefined values, DA compares with the retrieved values at host 1 , if the file is found and the price is comparable, it reserves the file and then moves to next host.

4. The process is repeated at the next host in the itinerary. After comparison, if the price is approximately same as the predefined value, DA reserves the file and sends cancellation request to previous host where DA finds the price little bit costly at the previous remote host
5. This process is repeated at all the hosts listed in the itinerary if and only if the file is present.

6. DA carries the information about the file to be downloaded back to the dispatching server and hand it over to User Agent.

7. The User agent is now responsible for displaying this information to the end user.

8. Finally, the end user decides to purchase the file and makes on-line payment to download the file to the user machine using DA.

\section{COMPARISON}

\subsection{Advantages}

1. During the course of file download procedure, after dispatching DA, even if the network is down for a while, the request can still be processed. The DA would have already reached the remote server and user request would have been acknowledged. The DA can be returned to the dispatching client along with the File/s, once the network is up. The user need not make fresh or repeated requests to obtain the same file/s.

2. DA can take network path which has less traffic thus increasing the speed of download. After obtaining the file/s requested, it can travel back in the predefined itinerary or can take another route which has less internet traffic. It can even be assigned the shortest path.

3. DA can be provided with the shortest itinerary to travel to the remote server which can reduce the number of hops.

4. If two or more DAs are sent to get multiple file requests, these DAs travel simultaneously, obtain files simultaneously and also travel back simultaneously.

5. The data size does not matter as the data reaches client along with mobile agents.

6. The time required to download multiple files using mobile agents is very less.

7. Multiple files from different servers can be downloaded simultaneously.

8. The time taken is reduced, speed is high and it is reliable as itinerary details give source and destination addresses. Constant monitoring of downloading process by the user is made easy.

9. The agent returns back with the files based on the older request when connection is reestablished and the same is handed over to WA.

10. User is relieved of repeated requests. He can continue with his other works.

11. Another important observation is that the speed of the internet does not affect mobile agents since they are assigned different itinerary to reach the server along the path where there is less traffic.

12. We have even tried by assigning shortest path to reach server which in turn has further reduced the round trip time of the mobile agents.

13. Figure 5 shows that, with increase in data size, client/ server model takes linear increase in the round trip time, whereas mobile agent technology saves time. By considering all the above said factors, practically proved that, the use of multiple mobile agents in downloading 
various types of files from remote servers is more efficient and faster.

\section{PSEUDO-CODES FOR VARIOUS AGENTS}

\subsection{Pseudo-Code for User Agent}

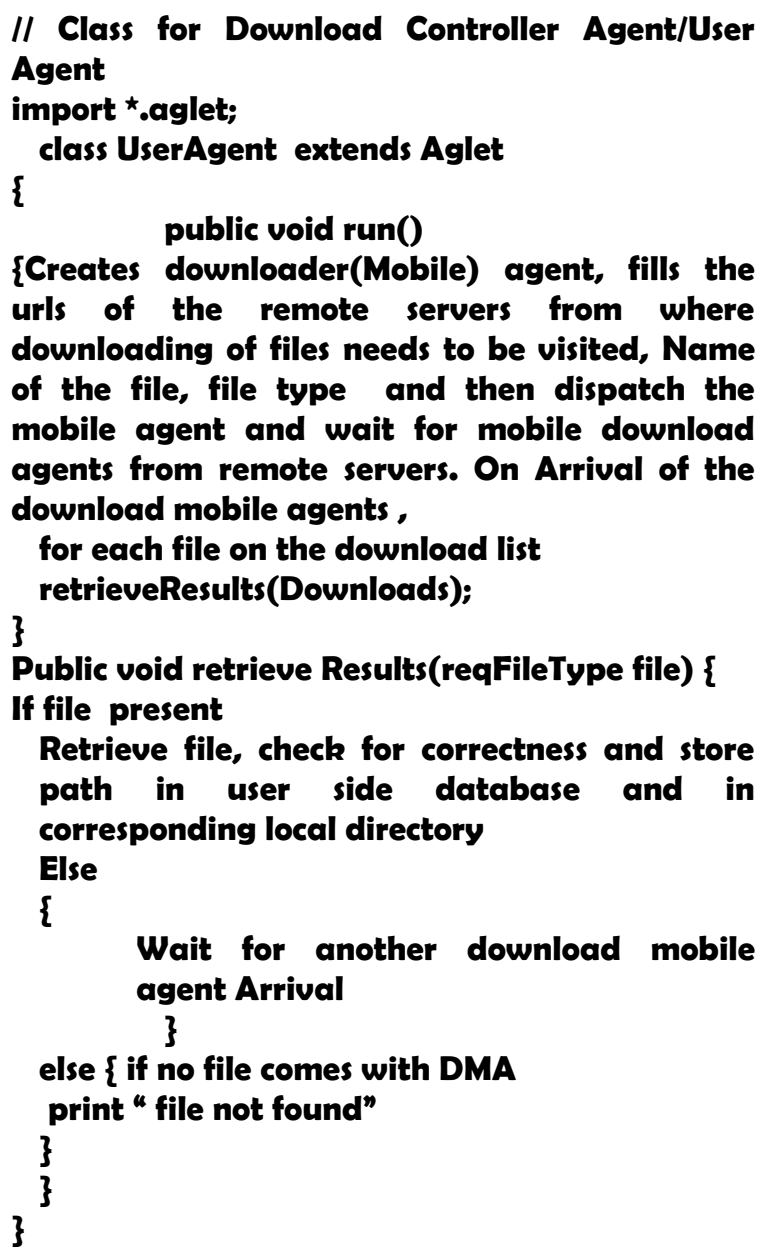

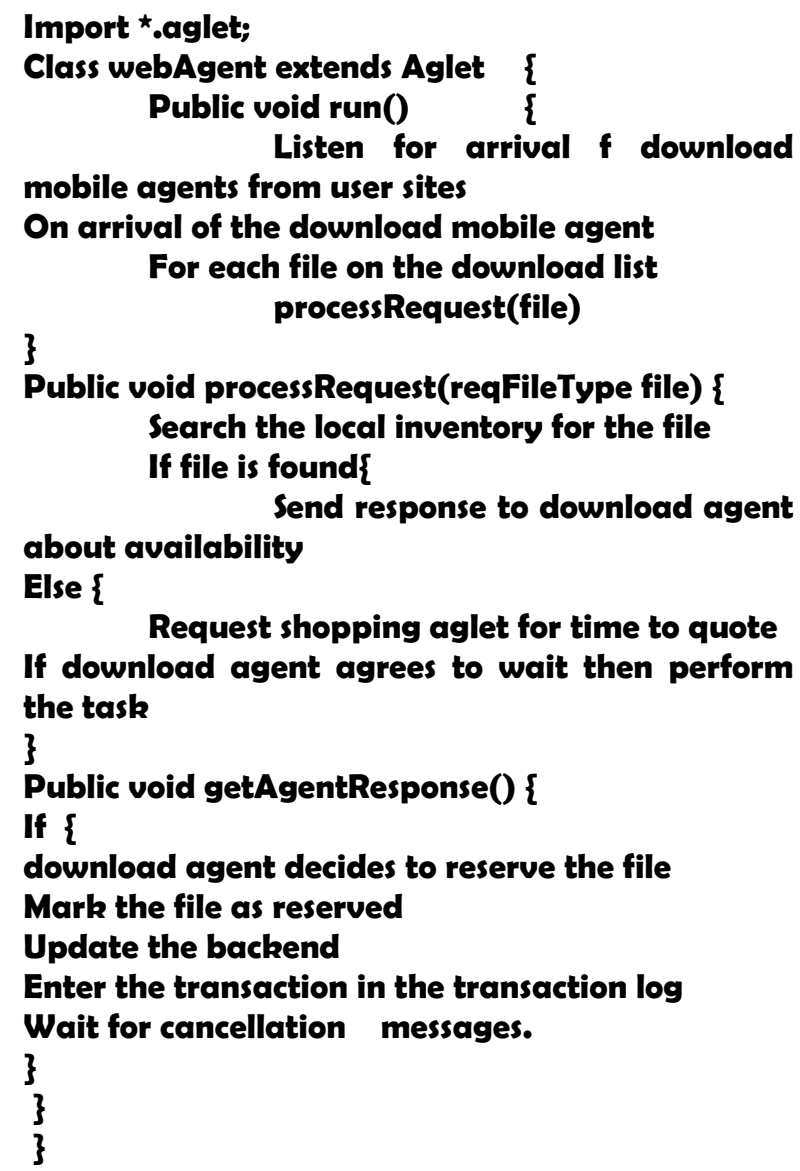

\section{CONCLUSION}

This paper introduces a practical mobile agent scenario which could be used for the purpose of the downloading of files from different hosts which is very much necessary for an e-commerce. Here the mobile agents help the buyers to download the necessary multimedia /files on-line. This also overcomes the problem of streaming that is very much necessary with $\mathrm{ftp}$ downloading of audio and video files. In case of the download is on paid basis, we have devised an algorithm which states that, the mobile agents are employed to reserve and cancel the files at different hosts depending upon the attribute comparison, which makes the user's task easy. This work also practically proves that it is more efficient than traditional ftp downloading. The one limitation of this work is Tahiti server must be enabled on all the hosts. 


\section{REFERENCE}

[1] Mobile Agents: Are they a good idea? Colin G. Harrison David M. Chess Aaron Kershenbaum

[2] Programming and Deploying Java ${ }^{\mathrm{TM}}$ Mobile Agents with Aglets, Danny B. Lange/ Mitsuru Oshima, Second Printing, Addison Wesley 1998

[3] David Kotz and Robert S. Gray. "Mobile Agents and the Future of the Internet", ACM Operating Systems Review, 33(3):7-13, August 1999.

[4] P. Dasgupta, N. Narasimhan, L.E. Moser and P.M. MelliarSmith, "MAgNET: Mobile Agents for Networked Electronic Trading ", IEEE Transactions on Knowledge and Data Engineering, Special Issue on Web Applications, July-August 1999.

[5] P. Dasgupta, N. Narasimhan, L.E. Moser and P.M. MelliarSmith, "A Supplier Driven Electronic Marketplace Using Mobile Agents", Proceedings of the First International Conference on Telecommunications and E-Commerce, Nashville, TN, Nov. 1998

[6] Danny B. Lange and Mitsuru Oshima, "Seven Good Reasons for Mobile Agents ", Communications of ACM, vol.42, no. 3, March 1999.

[7] Mrs. Nirmala C R, D. V Ramaswamy , Mrs. Jyothi N M"Performance Enhancement of E-Commerce Applications Using Mulitple Mobile Agents" World Academy of Science, Engineering and Technology, Volume 51, March 2009 ISSN 2070-3724, pp. 114-118

[8] Mrs. Nirmala C R, Dr. V Ramaswamy, Mrs. Jyothi N M Mobile Agents for Audio Search and Retrieval, International Journal of Computer Applications IJCA, March 2010, Volume 1, Article 15 ISSN 0975-8887.I

[9] A Beehive Approach to e-Commerce Mobile AgentsMartin van Hensbergen Bartek Gedrojc, Jan C.A. van der Lubbe.
Mrs. Nirmala C R , has obtained her B.E in Electronics and Communication discipline from Bapuji Institute of Engineering and Technology, Davangere. She has received her Masters Degree in Computer Engineering from reputed National Institute of Engineering., Surathkal in the year 2001. She has over sixteen years of teaching experience. She is currently working as Assistant Professor in Dept. of CS \&E, Bapuji Institute of Engineering \& Technology.

Right now she is pursuing her research work on "Applications of Multi Mobile Agents in E-Commerce" under the guidance of Dr. V.Ramaswamy, Professor \& Head, Department of Information Science \& Engineering, Bapuji Institute of Engineering \& Technology, Davangere

Dr. V Ramaswamy obtained his Ph.D degree from Madras university, in 1982, He is working as Professor and Head in the Department of Information Science and Engineering. He has more than 25 years of teaching experience including his four years of service in Malaysia. He is guiding many research scholars and has published many papers in national and international conference and in many international journals. $\mathrm{He}$ has visited many universities in USA and Malaysia.

Mrs. Jyothi N M, is currently working as lecturer in Dept. of MCA, Bapuji Institute of Engineering \& Technology, Davangere. She has her Masters Degree in Computer Applications completed in the year 2002 from Bharathidasan University, Trichy. She has five years of teaching and Five years of industry experience. 